\title{
Impact of a Novel Hospital-Based Opioid Stewardship Program on Nonsurgical Acute Pain Management in the Hospital
}

J Gen Intern Med 36(11):3608-10

DOI: $10.1007 / \mathrm{s} 11606-020-06404-\mathrm{x}$

(C) Society of General Internal Medicine 2021

\section{INTRODUCTION}

The opioid epidemic has resulted in efforts to prevent opioidrelated harms by improving opioid prescribing practices. Many patients are prescribed opioids for the first time in the hospital and over half of nonsurgical inpatients receive opioids for pain during their hospital stay. ${ }^{1,2}$ The dose and duration of initial opioid prescriptions has been shown to increase immediate and long-term opioid-related harms; therefore, inpatient prescribers have a crucial role in mitigating opioid-related risks for patients with pain. ${ }^{3,4}$

Multimodal pain management has been shown to decrease opioid exposure while effectively treating pain and current guidelines support its use in the hospital. ${ }^{5}$ This approach was not routinely utilized at New York-Presbyterian Lower Manhattan Hospital (LMH), an urban community hospital, suggesting the potential for unnecessary opioid prescribing and the associated increased risk of harm.

We created an opioid stewardship program (OSP) promoting prescriber education and pharmacy-led opioid stewardship rounds to increase the utilization of multimodal therapy (MMT) for acute inpatient nonsurgical pain. The program was modeled after hospital-based antibiotic stewardship programs (ASPs) which have proven efficacy utilizing education and in-person discussions. ${ }^{6}$ We hypothesized that increasing our adherence to MMT would provide effective pain control and decrease patient opioid exposure during hospitalization. The primary outcome was the percentage of hospitalized patients prescribed MMT for acute nonsurgical pain. Secondary outcomes included mean patient pain scores per hospital day, total opioid doses administered measured using milligram morphine equivalents (MME) per hospital stay, number of opioid prescriptions prescribed at discharge, and prescriber knowledge of MMT.

Received August 6, 2020

Accepted December 6, 2020

Published online January 19, 2021

\section{METHODS}

This quality improvement study included adults 18 years of age or older admitted to a hospital medicine service and prescribed intravenous or oral opioids for nonsurgical and noncancer pain no greater than 4 weeks duration. Data were manually abstracted from electronic health records (Allscripts ${ }^{\mathrm{TM}}$ ) during a 6month pre-intervention period and the 7-month intervention period. To compare pre- and post-intervention changes, Fisher's exact test was used for categorical characteristics and Wilcoxon signed-rank tests were used for continuous characteristics.

The intervention consisted of weekday pharmacy stewardship rounds using the existing model of our own ASP and scheduled education of prescribers, nursing, and physical therapy staff. OSP rounds included a face-to-face, phone, or secure messaging discussion regarding the pain regimen with the primary team.

\section{RESULTS}

The pre-intervention period included 179 patient-days and 48 unique patients with 50 encounters; the post-intervention period included 212 patient-days and 17 unique patients with 17 encounters. MMT utilization increased from $46 \%$ preintervention to $76 \%$ post-intervention $(P=0.029)$ (Fig. 1). There was no significant difference in mean reported pain scores in the first five days of hospitalization between the pre-intervention and post-intervention groups (Fig. 2). Mean overall opioid exposure per hospital stay decreased by $38 \%$ in the post-intervention group (153 vs. 99 MMEs per hospital stay; $P=0.29$ ) along with the number of patients prescribed opioids at discharge (13 vs. $6 ; P=0.52$ ). Provider knowledge of safe opioid prescribing practices assessed by survey was $96 \%$ pre-intervention and $98 \%$ post-intervention.

\section{DISCUSSION}

Implementing an OSP comprised of both structured provider education and opioid stewardship rounds increased the utilization of MMT by $30 \%$. Pain scores were similar in the preintervention and post-intervention groups while the amount of opioids prescribed during hospitalization and at discharge decreased post-intervention, suggesting that pain control was unchanged using MMT. Interestingly, provider knowledge of MMT and prescribing practices were discordant as less than 


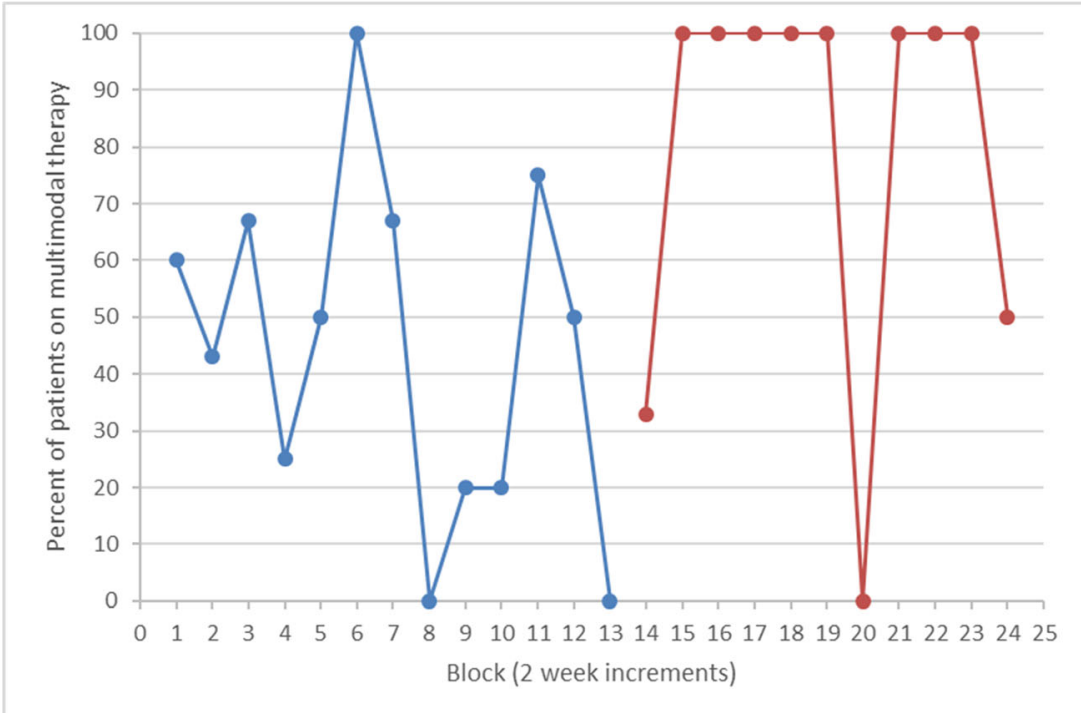

Figure 1 Utilization of multimodal therapy pre- and post-intervention. Each block represents two-week time increments. The pre-intervention period includes blocks 1-13 (blue) and the post-intervention period includes blocks 14-24 (red). Blocks with no patients meeting inclusion criteria were excluded from the run chart. In the pre-intervention group at weeks 8 and 13, there were $0 / 2$ patients prescribed multimodal therapy. At week 21 in the post-intervention group, there were 0/1 patients prescribed multimodal therapy. There were 4 weeks or 2 blocks between the end of the pre-intervention period and the start of the post-intervention period.

half of eligible patients received MMT in the pre-intervention group. Targeted patient-centered treatment discussions and education enabled prescribers to translate their knowledge into daily practice and increase utilization.

We believe our OSP was a feasible and effective intervention as the workflow integrated seamlessly with our preexisting antibiotic stewardship workflow. Capitalizing on one-on-one clinician guidance prevents electronic alert fatigue and addresses the individual clinical context not captured in systemwide or statewide restrictions.

Current pain management guidelines encourage providers to use MMT, but there is a gap in translation from knowledge to practice. This intervention provided effective decision support tools to help providers implement current best practices without oversimplifying the challenges of treating pain in the hospital. Future research is needed to study this intervention in

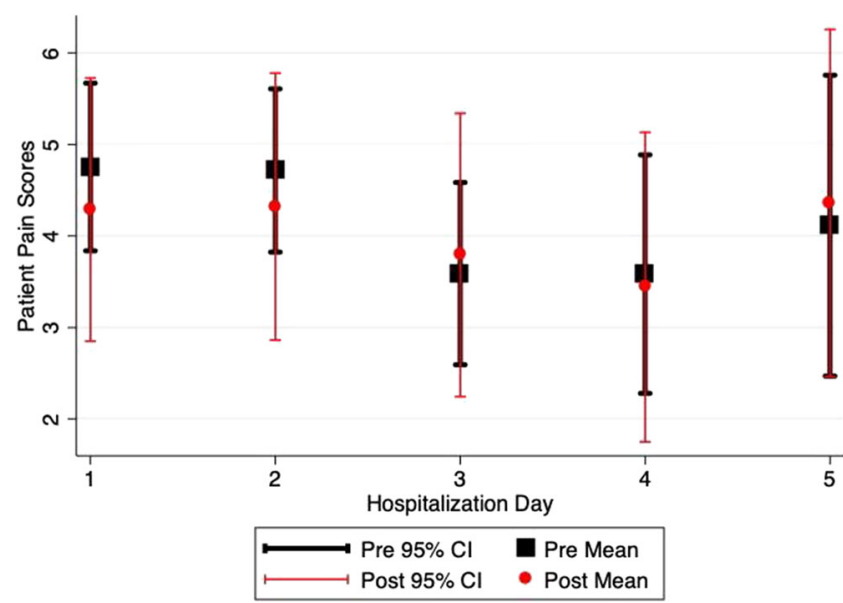

Figure 2 Pain scores pre- and post-intervention. other hospital settings such as larger institutions and academic medical centers.

Alexis Vien, $M D^{1,2}$

Grace Shyh, PharmD ${ }^{3}$

Deanna Jannat-Khah, $\mathrm{DrPH}, \mathrm{MSPH}^{4}$

Stephanie Chen, $M D^{2,5}$

Xiao Kuang, $R N^{6}$

Linda M. Gerber, $P H D^{7,8}$

Jennifer I. Lee, $M D^{1,2}$

${ }^{1}$ Division of General Internal Medicine, Weill Cornell Medicine,

New York, NY, USA

${ }^{2}$ New York-Presbyterian Weill Cornell Medical Center, New York, NY, USA

${ }^{3}$ Clinical Pharmacy, New York-Presbyterian Lower Manhattan Hospital,

New York, NY, USA

${ }^{4}$ Division of General Internal Medicine, Department of Rheumatology, Weill Cornell Medicine and Hospital for Special Surgery,

New York, NY, USA

${ }^{5}$ Department of Anesthesiology, Columbia University School of Medicine,

New York, NY, USA

${ }^{6}$ Department of Nursing, New York-Presbyterian Lower Manhattan Hospital,

New York, NY, USA

${ }^{7}$ Division of Nephrology and Hypertension, Weill Cornell Medicine,

New York, NY, USA

${ }^{8}$ Department of Healthcare Policy and Research, Division of Biostatistics and Epidemiology, Weill Cornell Medicine,

New York, NY, USA 
Corresponding Author: Alexis Vien, MD; Division of General Internal Medicine, Weill Cornell Medicine, New York, NY, USA (e-mail: ahw9001@med.cornell.edu).

\section{Compliance with Ethical Standards:}

Conflict of Interest: Deanna Jannat-Khah owns stock in Astrazeneca, Cytodyn, and Walgreens Boots Alliance. Otherwise the authors have no conflicts of interest to disclose.

\section{REFERENCES}

1. Herzig SJ, Rothberg MB, Cheung M, Ngo LH, Marcantonio ER. Opioid utilization and opioid-related adverse events in nonsurgical patients in US hospitals. J Hosp Med. 2014;9(2):73-81.

2. Calcaterra SL, Yamashita TE, Min S-J, Keniston A, Frank JW Binswanger IA. Opioid prescribing at hospital discharge contributes to chronic opioid use. J Gen Intern Med. 2016;31(5):478-485.

3. Deyo RA, Hallvik SE, Hildebran C, Marino M, Dexter E, Irvine JM, O'Kane N, Van Otterloo J, Wright DA, Leichtling G, Millet LM.
Association Between Initial Opioid Prescribing Patterns and Subsequent Long-Term Use Among Opioid-Naïve Patients: A Statewide Retrospective Cohort Study. J Gen Intern Med. 2017;32(1):21-27.

4. Hadlandsmyth K, Lund BC, Mosher HJ. Associations between initial opioid exposure and the likelihood for long-term use. J Am Pharm Assoc. 2019;59(1):17-22.

5. Herzig SJ, Calcaterra SL, Mosher HJ, Ronan MV, Groningen NV, Shek L, Loffredo A, Keller M, Jena AB, Nuckols TK. Safe opioid prescribing for acute noncancer pain in hospitalized adults: A systematic review of existing guidelines. J Hosp Med. 2018;13(4):256-262.

6. Dellit TH, Owens RC, McGowan JE, Gerding DN, Weinstein RA, Burke JP, Huskins WC, Paterson DL, Fishman NO, Carpenter CF, Brennan PJ, Billeter M, Hooton TM, Infectious Diseases Society of America, Society for Healthcare Epidemiology of America. Infectious Diseases Society of America and the Society for Healthcare Epidemiology of America guidelines for developing an institutional program to enhance antimicrobial stewardship. Clin Infect Dis. 2007;44(2):159-177.

Publisher's Note: Springer Nature remains neutral with regard to jurisdictional claims in published maps and institutional affiliations. 